\title{
Treat-to-target Endpoint Definitions in Systemic Lupus Erythematosus: More Is Less?
}
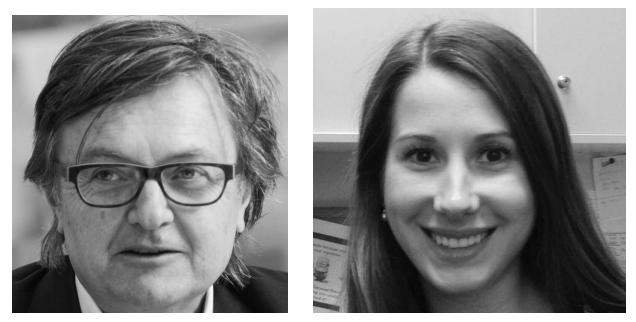

The use of treat-to-target (T2T) strategies has revolutionized the treatment of rheumatoid arthritis (RA) and other chronic diseases such as hypertension and diabetes. Evidence that attainment of a prespecified endpoint is associated with improved longterm outcomes dates to the TICORA study of 2003, which used a prespecified definition of low disease activity ${ }^{1}$. From that auspicious beginning of T2T in rheumatology, we have now come full circle. For example, the American College of Rheumatology (ACR)/European League Against Rheumatism definition of remission in RA was grounded on empirical analysis of factors contributing to improved outcomes ${ }^{2}$. These prospectively derived and comprehensively validated endpoints form the basis of RA treatment guidelines that are now routinely applied in clinical practice $^{3}$, contributing to a transformation in outcomes for patients with RA that includes significant improvements in survival ${ }^{4}$.

Sadly, in systemic lupus erythematosus (SLE) the same story cannot yet be told. In the same 2 decades in which profound mortality improvements in RA were observed ${ }^{4}$, there has been virtually no improvement in mortality in $\mathrm{SLE}^{5}$, and only 1 novel target therapy has been approved ${ }^{6}$. Many trials of targeted therapies have been done, but have failed; reasons for the shortage of breakthrough medicines for SLE include the clinical and biological heterogeneity of the disease. However, a lack of well-validated endpoints is certainly a contributory factor to the recurrent failure of clinical trials in SLE; a report in January 2019 in Nature Biotechnology ${ }^{7}$ highlighted the lack of validated endpoints for trials in SLE as a "crisis." T2T studies such as those that have shaped the management of RA are difficult to design in the absence of powerful new medicines to place in a treatment strategy, although it is worth noting that the first successful T2T strategy trials in RA used conventional disease-modifying antirheumatic drugs ${ }^{1}$. In SLE, there is a circular problem, in that the lack of endpoints stifles the demonstration of drug efficacy, and the lack of new drugs prevents the execution of treatment strategy trials.

In short, empirically validated endpoints for SLE research and eventual translation to practice are acutely needed, and to this end momentum has been building in recent years. Prompted by 2 important reviews ${ }^{8,9}$, a series of multinational processes has led to the proposal of new T2T definitions of low disease activity [the Lupus Low Disease Activity State $\left(\right.$ LLDAS $^{10}$ ) and remission ${ }^{11}$ ] that are now the subjects of multiple validation studies and have been successfully tested in trials. Although the results of prospective studies to once and for all evaluate LLDAS and remission for SLE are eagerly awaited, encouraging data are emerging from retrospective analysis of large cohorts. After initial demonstration in a single-center cohort that LLDAS attainment was associated with protection from flare and irreversible organ damage accrual ${ }^{10}$, studies followed that showed that LLDAS attainment is associated with improved health-related quality of life, and has superior performance characteristics to expert opinion unguided by a definition ${ }^{12,13}$. Importantly, the finding that attainment of LLDAS is associated with protection from organ damage accrual has been repeated in multiple other independent cohorts, in Europe and North America $^{14,15,16,17,18}$, with a remarkably consistent finding that $50 \%$ of observed time in LLDAS results in a $50 \%$ reduction in damage accrual. The most intensive evaluation of Definition of Remission in SLE (DORIS) ${ }^{11}$ so far was published by Petri and Magder in 2018, wherein it was demonstrated that remission attainment was highly protective from damage accrual, and that this protection was achieved at a lower exposure than was the case for LLDAS $^{15}$. This suggests that the metrologically appealing goal of deeper protection from adverse outcome with concentrically more stringent outcome definitions ${ }^{19}$ is at hand.

It is in this context that the study presented by Ugarte-Gil, et al in this issue of The Journal ${ }^{20}$ should be considered. Using a subset of the large and well-established GLADEL cohort of adult patients with SLE, the authors reviewed the outcomes of 902 mostly young females who had suboptimal disease control at the time of entry to the registry, to evaluate factors associated with certain outcome states. In this paper,

See Predictors of improvement in SLE, page 1299

Personal non-commercial use only. The Journal of Rheumatology Copyright @ 2019 . All rights reserved. 
those states were defined as "remission" [Systemic Lupus Erythematosus Disease Activity Index $($ SLEDAI $)=0$, prednisolone dose $<5 \mathrm{mg} /$ day, immunosuppressants allowed] and "low disease activity state" (SLEDAI < 4, prednisolone dose $<7.5 \mathrm{mg}$ /day, immunosuppressants allowed). Importantly, these definitions diverge from the increasingly well-validated LLDAS and DORIS remission definitions ${ }^{10,11}$. The new findings show that baseline higher disease activity, mucocutaneous, or renal involvement made attainment of either endpoint less likely, with remission also less likely in patients with baseline hematological manifestations or prior immunosuppressant use. Data such as these may in the future guide physicians in determining which patients may benefit from more intensive control of disease activity, albeit without escalation of glucocorticoids, which independently contribute to damage accrual risk in $\mathrm{SLE}^{21}$. This study follows an earlier one from the same authors, in which it was shown that attainment of these states was each associated with significant protection from permanent organ damage as measured by the SLICC-ACR damage index ${ }^{22}$.

The value of this cohort, and the quality of the data, analysis, and conclusions in these studies are not in doubt. However, it may be prudent to flag a concern about introducing new SLE endpoint definitions, especially when defined based on data from preestablished cohorts. LLDAS is defined incorporating similar variables to those described in Ugarte-Gil, et $a l^{20}$ with increased stringency through 3 additional criteria: the exclusion of major organ disease activity and of new-onset activity compared to the last visit, and the inclusion of a physician's global assessment $<1(0-3$ scale), ensuring exclusion of activity that may otherwise be missed on the SLEDAI-2K. The DORIS ${ }^{11}$ framework for remission definitions in SLE is based around a clinical SLEDAI $=0$ and PGA $<0.5$ (0-3 scale), with varying allowance for serology, prednisolone $<5 \mathrm{mg} /$ day and immunosuppression use; some 8 possible definitions of remission arise from this framework. The low disease activity and remission definitions used in Ugarte-Gil, et $a l^{20}$ meaningfully differ from LLDAS and DORIS remission.

The LLDAS and DORIS remission definitions were determined a priori, based on consensus processes involving multinational expert panels convened specifically for this purpose, and without limitation by variables existing in specific cohort datasets; these definitions were subsequently tested in an extensive range of validation studies. In contrast to studies that test an endpoint that had been designed $a$ priori, Ugarte-Gil, et al have tested endpoint definitions constructed from the variables collected in an existing dataset. This constrains the choice of concepts included in the definitions of low disease activity and remission, and leaves open the question of face and content validity. While their findings ${ }^{20,22}$ are broadly consistent with studies of LLDAS and DORIS remission, posthoc assignment of endpoint definitions, driven by the data available, remains a significant limitation. This methodological concern is joined by another, perhaps more sociological one. While exploration of the nuances of endpoint definitions may be of interest to SLE measurement researchers, when definitions of such key concepts as low disease activity and remission shift from study to study, it is potentially confusing to other rheumatologists and even more so to the wider medical community and to industry. Adding more endpoint definitions to the landscape, other than in studies designed to push toward measurably superior endpoint performance, may have a downside; the aforementioned "crisis" in SLE endpoints for clinical trials ${ }^{7}$ is real, and a time when industry quits SLE research is not beyond imagining. Thus, we wonder whether, regarding the number of SLE treatment endpoint definitions in use, less might actually be more.

ERIC F. MORAND D, MBBS(Hons), FRACP, PhD, Head, School of Clinical Sciences, Monash University; Head of Rheumatology, Monash Health;

VERA GOLDER (D), MBBS(Hons), BMedSc(Hons), FRACP, Rheumatologist, Monash Health, Melbourne, Australia.

Address correspondence to E.F. Morand, Monash Medical Centre, 246 Clayton Road, Clayton 3168, Melbourne, Australia. E-mail:

eric.morand@monash.edu

\section{REFERENCES}

1. Grigor C, Capell H, Stirling A, McMahon AD, Lock P, Vallance R, et al. Effect of a treatment strategy of tight control for rheumatoid arthritis (the TICORA study): a single-blind randomised controlled trial. Lancet 2004;364:263-9.

2. Felson DT, Smolen JS, Wells G, Zhang B, van Tuyl LH, Funovits J, et al. American College of Rheumatology/European League against Rheumatism provisional definition of remission in rheumatoid arthritis for clinical trials. Ann Rheum Dis 2011;70:404-13.

3. Smolen JS, Landewe R, Bijlsma J, Burmester G, Chatzidionysiou K, Dougados M, et al. EULAR recommendations for the management of rheumatoid arthritis with synthetic and biological diseasemodifying antirheumatic drugs: 2016 update. Ann Rheum Dis 2017;76:960-77.

4. Zhang Y, Lu N, Peloquin C, Dubreuil M, Neogi T, Avina-Zubieta JA, et al. Improved survival in rheumatoid arthritis: a general population-based cohort study. Ann Rheum Dis 2017;76:408-13.

5. Jorge AM, Lu N, Zhang Y, Rai SK, Choi HK. Unchanging premature mortality trends in systemic lupus erythematosus: a general population-based study (1999-2014). Rheumatology 2018:57:337-44.

6. Wallace DJ. The evolution of drug discovery in SLE. Nat Rev Rheumatol 2015;11:616-20.

7. Dolgin E. Lupus in crisis: as failures pile up, clinicians call for new tools. Nat Biotechnol 2019;37:7-8.

8. Franklyn K, Hoi A, Nikpour M, Morand EF. The need to define treatment goals for systemic lupus erythematosus. Nat Rev Rheumatol 2014;10:567-71.

9. van Vollenhoven RF, Mosca M, Bertsias G, Isenberg D, Kuhn A, Lerstrom K, et al. Treat-to-target in systemic lupus erythematosus: recommendations from an international task force. Ann Rheum Dis 2014;73:958-67.

10. Franklyn K, Lau CS, Navarra SV, Louthrenoo W, Lateef A, Hamijoyo L, et al. Definition and initial validation of a Lupus Low Disease Activity State (LLDAS). Ann Rheum Dis 2016;75:1615-21.

Personal non-commercial use only. The Journal of Rheumatology Copyright (c) 2019. All rights reserved. 
11. van Vollenhoven R, Voskuyl A, Bertsias G, Aranow C, Aringer M, Arnaud L, et al. A framework for remission in SLE: consensus findings from a large international task force on definitions of remission in SLE (DORIS). Ann Rheum Dis 2017;76:554-61.

12. Golder V, Kandane-Rathnayake R, Hoi AY, Huq M, Louthrenoo W, An Y, et al. Association of the lupus low disease activity state (LLDAS) with health-related quality of life in a multinational prospective study. Arthritis Res Ther 2017;19:62.

13. Golder V, Huq M, Franklyn K, Calderone A, Lateef A, Lau CS, et al. Does expert opinion match the operational definition of the Lupus Low Disease Activity State (LLDAS)? A case-based construct validity study. Semin Arthritis Rheum 2017;46:798-803.

14. Zen M, Iaccarino L, Gatto M, Saccon F, Larosa M, Ghirardello A, et al. Lupus low disease activity state is associated with a decrease in damage progression in Caucasian patients with SLE, but overlaps with remission. Ann Rheum Dis 2018;77:104-10.

15. Petri M, Magder LS. Comparison of remission and lupus low disease activity state in damage prevention in a United States systemic lupus erythematosus cohort. Arthritis Rheumatol 2018;70:1790-5.

16. Fanouriakis A, Adamichou C, Koutsoviti S, Panopoulos S, Staveri $\mathrm{C}, \mathrm{Klagou} \mathrm{A}$, et al. Low disease activity-irrespective of serologic status at baseline-associated with reduction of corticosteroid dose and number of flares in patients with systemic lupus erythematosus treated with belimumab: A real-life observational study. Semin Arthritis Rheum 2018;48:467-74.
17. Tsang-A-Sjoe MW, Bultink IE, Heslinga M, Voskuyl AE. Both prolonged remission and Lupus Low Disease Activity State are associated with reduced damage accrual in systemic lupus erythematosus. Rheumatology 2017;56:121-8.

18. Piga M, Floris A, Cappellazzo G, Chessa E, Congia M, Mathieu A, et al. Failure to achieve lupus low disease activity state (LLDAS) six months after diagnosis is associated with early damage accrual in Caucasian patients with systemic lupus erythematosus. Arthritis Res Ther 2017;19:247.

19. Morand EF. Connective tissue diseases: Remission in SLE - are we there yet? Nat Rev Rheumatol 2016;12:696-8.

20. Ugarte-Gil MF, Wojdyla D, Pons-Estel GJ, Quintana R, Gómez Puerta J, Catoggio LJ, et al. Predictors of remission and low disease activity state in systemic lupus erythematosus: data from a multiethnic, multinational Latin-American lupus cohort. J Rheumatol 2019;46:1299-1308

21. Apostolopoulos D, Morand EF. It hasn't gone away: the problem of glucocorticoid use in lupus remains. Rheumatology 2017; $56: 1114-22$.

22. Ugarte-Gil MF, Wojdyla D, Pons-Estel GJ, Catoggio LJ, Drenkard $\mathrm{C}$, Sarano J, et al. Remission and low disease activity status (LDAS) protect lupus patients from damage occurrence: data from a multiethnic, multinational Latin American Lupus Cohort (GLADEL). Ann Rheum Dis 2017;76:2071-4.

J Rheumatol 2019;46:1256-8; doi:10.3899/jrheum.190223 